\title{
Design and Implementation of a Long Range Indoor VLC System using PWM
}

\author{
Majed Basha ${ }^{1,},{ }^{*}$ Martin J. Sibley ${ }^{1}$ and Peter J. Mather ${ }^{1}$ \\ ${ }^{1}$ School of Computing and Engineering, University of Huddersfield, United Kingdom \\ majed.basha@hud.ac.uk; m.j.n.sibley@hud.ac.uk; p.j.mather@hud.ac.uk \\ *Correspondence: majed.basha@hud.ac.uk
}

Received: 25 $5^{\text {th }}$ November 2018; Accepted: 19 ${ }^{\text {th }}$ December 2018; Published: $1^{\text {st }}$ January 2019

\begin{abstract}
Visible Light Communications (VLC) is currently considered one of the most promising Optical Wireless Communications (OWC) for commercial applications, due to the widespread deployment of Light Emitting Diodes (LEDs) for energy efficiency, durability and low cost. With the ability to provide several THz of bandwidth, VLC is expected to co-exist with legacy and future Radio Frequency (RF) media as a reliable solution to the rapid demand of high-speed wireless communication. VLC is challenged by two main drawbacks: ambient light interference and the transmission range. To overcome these drawbacks, we propose a tuned VLC Analog Front-End (AFE) using simple and low-cost electronic circuity. The proposed VLC receiver architecture consists of a transimpedance amplifier, a fourth order Multiple Feed Back (MFB) band pass filter and a comparator. Experimental results demonstrated that by employing the proposed front-end the VLC transmission range could be extended up to $4.7 \mathrm{~m}$ by using Pulse Width Modulation (PWM). Moreover, the proposed system showed robustness against ambient light interference under the indoor scenario.
\end{abstract}

Keywords: VLC; Pulse Width Modulation; LEDs; Multiple Feedback; SNR

\section{Introduction}

VLC have been attracting researcher's attention through the last decade as a reliable solution to the rapid demand of high-speed wireless communications. The idea of VLC systems is based on the dual use of LEDs for both illumination and data communications [1]. The current radio frequency (RF) wireless spectrum below $6 \mathrm{GHz}$ is rapidly running out of bandwidth for high data-rate communication therefore, leading to spectrum congestion which means lack of sufficient bandwidth to be shared among a large number of users [2]. Furthermore, RF wireless communication suffer from several drawbacks such as expensive licensing, security issues and high installation cost in addition to multipath propagation effects in urban environments which reduces its efficiency [3]. Based on these drawbacks, an alternative technology is needed to enable continuous indoor wireless communication with the required data rates and increasing demand of users. With an un licensed bandwidth of $\sim 300 \mathrm{THz}$, VLC offer a reliable solution which can overcome these restrictions. VLC offers superior features over RF including low-cost with no licensing fee, high bandwidth, high security due to non-interference with electromagnetic waves and energy efficiency. VLC takes full advantage of the existing visible Light-emitting diodes (LEDs) infrastructure offering the dual use of

Majed Basha, Martin J. Sibley and Peter J. Mather “Design and Implementation of a Long Range Indoor VLC System using PWM", Annals of Emerging Technologies in Computing (AETiC), Print ISSN: 2516-0281, Online ISSN: 2516-029X, pp. 20-27, Vol. 3, No. 1, $1^{\text {st }}$ January 2019, Published by International Association of Educators and Researchers (IAER), DOI: 10.33166/AETiC.2019.01.003, Available: http://aetic.theiaer.org/archive/v3/v3n1/p3.html. 
illumination and high-speed data communication. VLC utilizes visible light which is totally harmless to humans and environment therefore is considered as a sustainable and green technology which will take us a step forward towards "green communication" [4]. The particular characteristics of VLC systems, enables it to provide solutions for a broad spectrum of applications, including wireless local area networks (WLAN), indoor localization and positioning systems where current Global positioning system (GPS) is not available, Intelligent transportation systems (ITS), and under water communication offering a range of data rates from a few Kbps up to 10Gbps [5].

An anti-low frequency OOK- based VLC receiver was implemented in [6] which has the ability to reduce indoor low frequency light noise however its transmission range was limited to $1 \mathrm{~m}$ distance. Another study proposed analog filter design which employed a DC offset removal circuit and a band stop filter to remove indoor fluorescent light interference [7].

The main idea of the research is increasing the receiver sensitivity by employing an analog filtering stage within the receiver front-end while the transmission is carried out using a low-power commercial white light LED, enabling the system to provide both illumination and digital communication. The proposed VLC front-end employs a high gain, high Quality (Q) factor band pass filter with the Multiple Feed Back (MFB) topology, which operates at a defined center frequency range. The proposed MFB active filter uses the gain of the operational amplifier at the defined transmission frequency maximizing the gain and therefore extending the transmission range. The band pass filter behaves as a resonant circuit which detects the desired signal at the transmitting center frequency and rejects unwanted frequencies including ambient light noise frequencies [8]. The modulation scheme used through this work was Pulse Width Modulation (PWM).

\section{Channel characteristics for indoor LOS propagation model}

To design, implement and operate an efficient optical wireless communication system, it is crucial to understand the channel characteristics. Optical power loss due to channel characteristics depends mainly on path loss and multipath dispersion [9]. The two main configurations considered in VLC are direct LOS and non-LOS configurations. In LOS configuration the reflected light on different surfaces is not taken in consideration, while in non-LOS configuration which is also known as (diffuse channel) the received signal is considered with the reflections of light on different surfaces in the indoor environment. These reflections may cause multipath distortion making it more complex to estimate the path loss model [10]. In this thesis work direct -LOS configuration will be considered.

In indoor VLC systems, the signal is transmitted using an LED and detected photodiodes. In this case, the Lambertian radiant intensity model. The angular distribution of the intensity model can be expressed as:

$$
R_{o}(\emptyset)=\left\{\begin{array}{cl}
\frac{(m+1)}{2 \pi} \cos ^{m}(\varnothing) & \text { for } \emptyset=[-\pi / 2, \pi / 2] \\
0, & \text { for } \emptyset \geq \pi / 2
\end{array}\right.
$$

Where $m$ is the Lambert's mode number and $\phi$ is the angle of irradiance. The Lambertian order $m$ is also related to the LED semi-angle at half power $\emptyset_{1 / 2}$ as seen in (2).

$$
m=\frac{-\ln 2}{\ln \left(\cos \emptyset_{1 / 2}\right)}
$$

In short range LOS when both the transmitter and receiver are aligned, the light absorption and scattering is too low and can be ignored. In this case the DC channel gain $H_{\text {los }}$ can be approximated as:

$$
H_{\text {los }}=\left\{\begin{array}{c}
\frac{A_{r}(m+1)}{2 \pi d^{2}} \cos ^{m}(\varnothing) T_{S}(\Psi) \cos (\Psi), 0 \leq \Psi \leq \Psi_{c} \\
0,
\end{array}\right.
$$

Where $A_{r}$ is the area of the detector, $d$ is the transmission distance, $m$ is the order of Lambertian emission, $\phi$ is the angle of irradiance, $\Psi$ is the receiver angle of incidence, $\Psi_{c}$ is the receivers' field of view (FOV), and $T_{s}(\Psi)$ is the transmission response of the optical system. According to (4) the channel gain is inversely proportional to the transmission distance [11]. 
In longer distance VLC, the transmitted signal will be affected by atmosphere absorption and light scattering, which deeply reduces the channel gain. The monolithic channel gain due to attenuation of light intensity $H_{\text {owc }}$ can be defined as:

$$
\begin{aligned}
& H_{o w c}=H_{l o s}(\lambda) \exp (-\beta(\lambda) \cdot d) \\
& \beta(\lambda)=\alpha_{m}+\alpha_{a}+\sigma_{m}+\sigma_{a}
\end{aligned}
$$

Where $\beta(\lambda)$ is the attenuation coefficient, $\alpha_{m}$ is the atmospheric absorption coefficient, $\alpha_{a}$ is the absorption coefficient of aerosol particles, $\sigma_{m}$ is the scattering coefficient of atmospheric molecules, and $\sigma_{a}$ is the scattering coefficient of aerosol particles [12]. From the above equations, we can conclude that by increasing the transmission distance, the channel gain will decrease, which means that long-range VLC applications will suffer from low SNR. Therefore, to achieve longer transmission distance, higher transmission power and higher receiver sensitivity are needed which is challenging in practical VLC applications.

\section{VLC system design and parameters}

Figure 1. shows the block diagram of the proposed VLC system. The transmitter (Tx) is comprised of a signal generator and the LED driver circuit. The data generated by the signal generator will be encoded and transmitted by the diver circuit using Manchester encoding. Next, the data is transmitted by visible light, which is emitted by white light LED. At the receiver (Rx) side the signal is detected by the photodiode. Due to the communication channel characteristics, the data received at the Rx side is faded, attenuated, and interfered with ambient light noise. In order to reduce the noise interference and increase the receiver sensitivity we propose a receiver design, which is comprised of a Transimpedance Amplifier (TIA) and a fourth order band pass filter with MFB topology. Finally, the received signal is observed and analyzed through the oscilloscope.

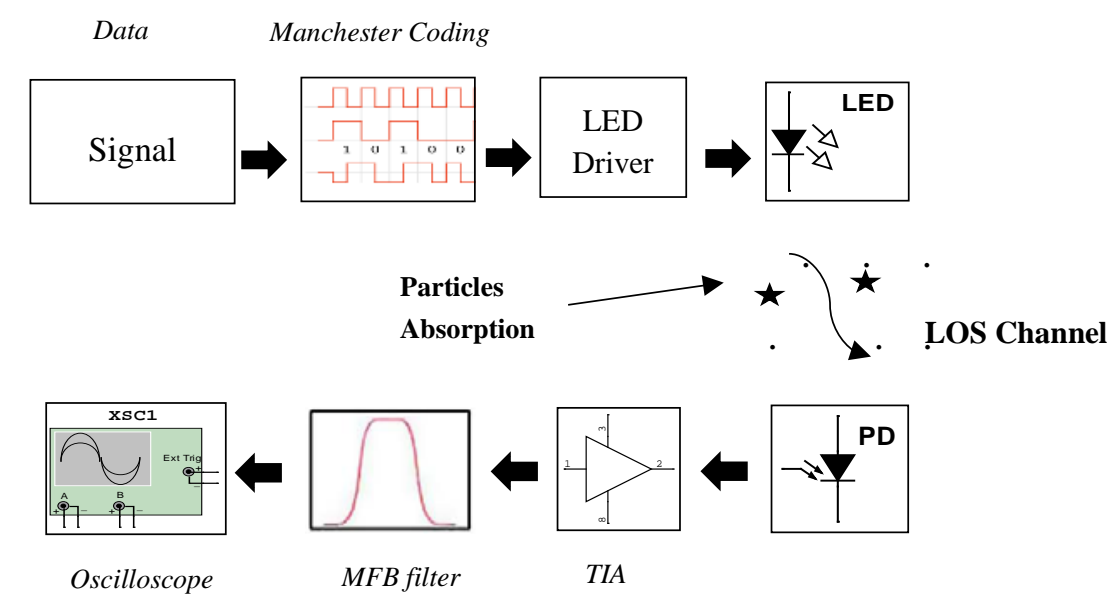

Figure 1. VLC system Block Diagram.

\subsection{VLC platform parameters and specifications}

The main goal of the experiments is to improve the transmission range of a Visible Light Communication (VLC) system using a low power white light LED for transmission within the indoor lab environment under the influence of fluorescent lights. The electronic components were carefully chosen to meet the system requirements. A low cost commercial white light Nichia (NSDL570GS-K1) 0.2 Watts power LED with half power angle of 70 degrees, biased at $100 \mathrm{~mA}$ was chosen. Nichia LEDs have the advantages of durability, homogeneity and brightness, which makes it suitable for dual purpose of illumination in addition to data communication. The LED relative radiant intensity peaks at the wave length of 590nm. At the receiver, the chosen photodiode was the VTB8440BH Silicon photodiode with infrared rejection filter to mitigate the effect of ambient interference. The photodiode has a spectral response, which is only influenced by visible light, making it suitable for this application. This photodiode also has the advantages of high speed and low noise due to its low 
junction capacitance and low dark current. The Op-amp used for the Transimpedance amplifier and the active filter was the LT1056 JFET input operational amplifier. This Op-amp was chosen with a gain bandwidth product of $6.5 \mathrm{MHz}$ due to the Multiple Feedback (MFB) minimum gain bandwidth product requirement, so that the filter will not oscillate. The LT1056 Op-amp is also suitable to be used as a photodiode amplifier due to its low input bias current.

\section{Design and implementation of a Long-Range VLC system using PWM}

PWM have been widely used in VLC systems including LED dimming control applications. By using PWM, the dimming level of the LED could be controlled without affecting the transmitted data. As the pulse amplitude is constant in PWM therefore, the spectrum of the light emitted by LED remains constant, and by the variation of the pulse width, the LED dimming level could be controlled [13]. In addition, to avoid flickering the duty ratio could be adjusted to a certain percentage without affecting the transmitted data. In some VLC applications high data rates is not required such as text delivery shopping, digital signage and running text applications. Thus, in these cases using complex and expensive modulation is wasteful. In this section, a VLC system for long-range transmission using PWM will be designed and implemented. Fig 2. shows the experimental setup of the VLC system based on PWM. The proposed VLC system is comprised of a function generator, which generated a pulse signal of a chosen frequency. PRBS data stream is internally modulated using PWM and transmitted through the LED. The proposed receiver circuit detects the received signal and the captured signal is analyzed by the oscilloscope.

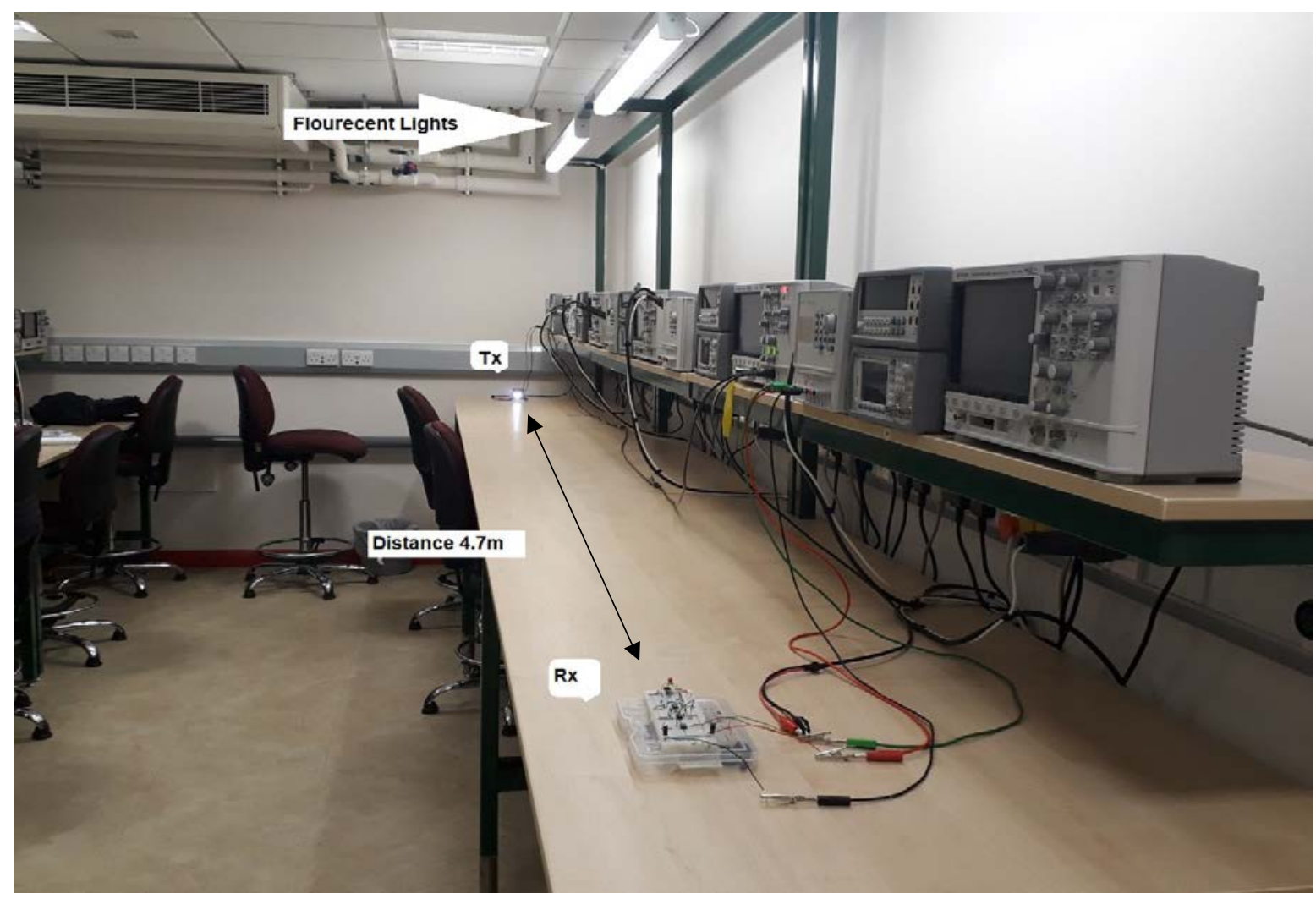

Figure 2. Experimental setup of long-range VLC system based on PWM

\subsection{LED driver circuit design}

The LED driver circuit is comprised of a low power LED $0.2 \mathrm{~W}$ driven by a common collector configuration (CC) as shown in Fig LED. The high input impedance and low output impedance with an approximate unity gain of the CC configuration makes it a good choice as a buffer in the transmitter design [14]. The resistors $R_{1}$ and $R_{2}$ form a voltage divider that controls the base emitter 
voltage. The coupling capacitor $C_{1}$ blocks any DC signal at the input. The resistor $R_{3}$ controls the LED forward current and $R_{4}$ degenerates the emitter for early voltage improvement.

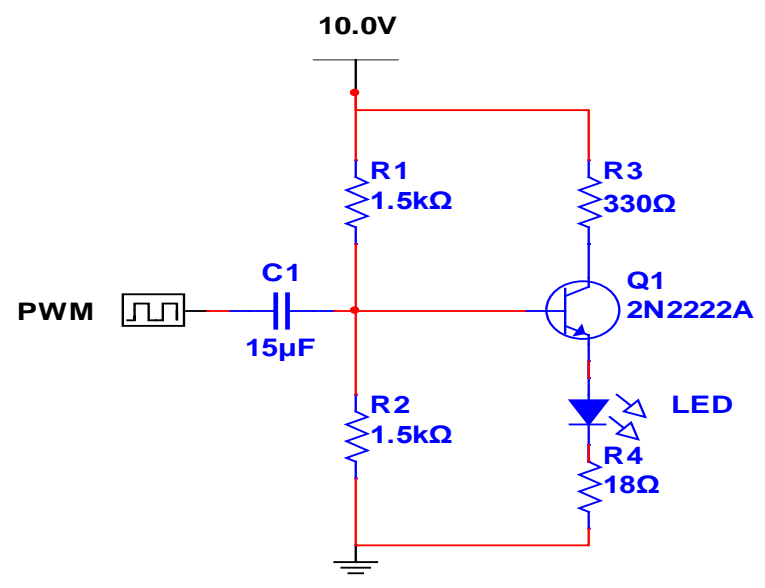

Figure 3. LED Driver Circuit

\subsection{VLC Receiver Design}

The proposed receiver circuit is comprised of a photodiode, Transimpedance amplifier, $4^{\text {th }}$ order multiple feedback band pass filter and a comparator. Fig 4. shows the proposed VLC receiver schematic diagram with the value of the components corresponding to each module. After being detected by the photodiode, the obtained electrical signal is fed to the TIA where the current to voltage conversion occurs. The proposed $4^{\text {th }}$ order multiple feedback band pass filter operates at the defined transmission frequency allowing only the desired frequency band and rejecting unwanted frequencies including ambient light interference caused by fluorescent lamps and other lighting backgrounds within the lab environment. Finally, to overcome the signal distortion the signal is fed to a comparator for recovery.

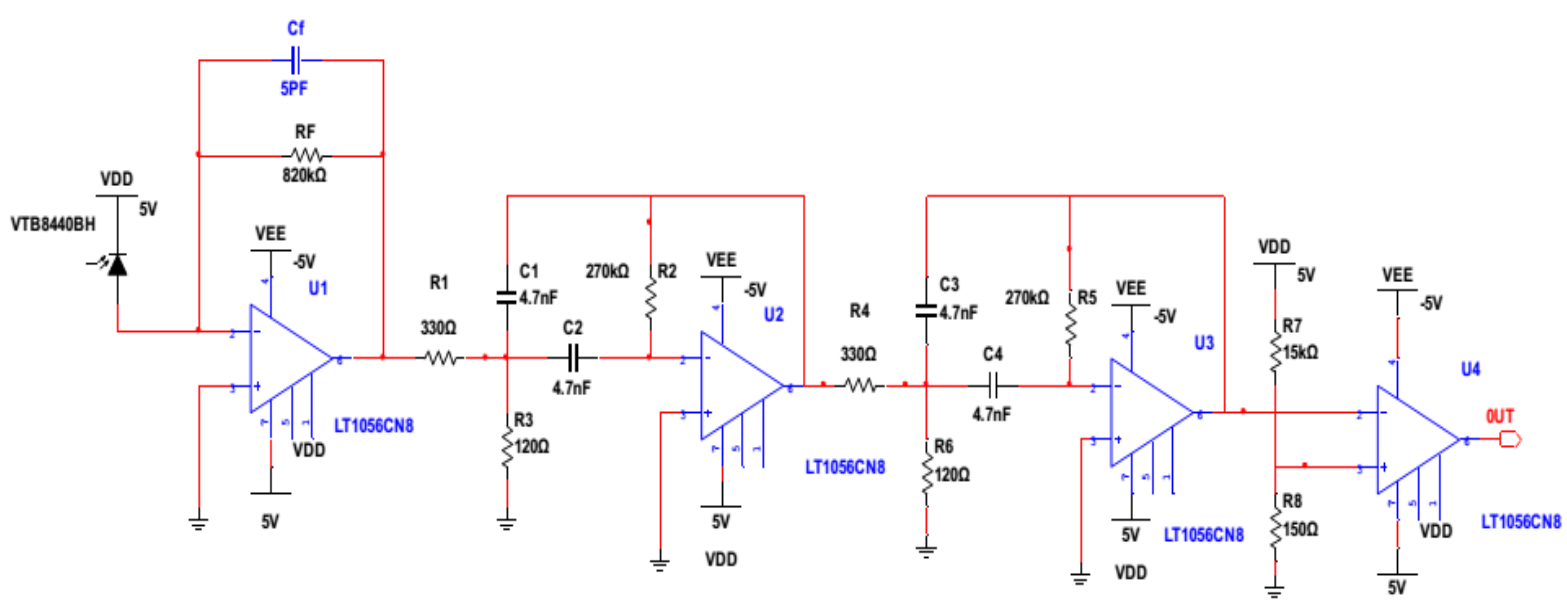

Figure 4. Schematic diagram of the proposed receiver circuit.

\section{Results and Analysis}

Experimental tests were performed to measure the performance of the proposed VLC system for the indoor condition with the availability of florescent lights and noise background. The first approach was to set the transmitter side. Using function generator, a pulse signal with frequency of $5 \mathrm{kHz}$ is generated. A pulse width of $100 \mu$ s and edge time of $50 \mathrm{~ns}$ was chosen. A PRBS signal with a bit rate of $10 \mathrm{Kbps}$ was modulated using PWM and width deviation of $20 \mu \mathrm{s}$. In the receiver side, the MFB band pass filter is designed to operate at the transmission frequency which is $5 \mathrm{kHz}$. 


\subsection{System improvement by employing the MFB band pass filtering stage}

To measure the efficiency of the proposed receiver the pulse shape of the signal captured by the oscilloscope is observed and compared to the transmitted signal with and without the filtering stage at varied distances. The comparator is then adjusted to the required threshold voltage at the maximum achievable distance to correct the pulse shape. Fig 5. shows the received signal at the output of the TIA without filtering at distances of $1.5 \mathrm{~m}$, the effect of noise caused by ambient light on the received pulse could be clearly seen, in addition the receiver detection range is limited to $1.5 \mathrm{~m}$ and further than this distance no signal can be detected. To measure the improvement achieved by adding the filtering stage the received signal is observed at varied distances and signal to noise ratio is evaluated with and without filtering. Fig 6 . shows the received signal at the distance of $4.7 \mathrm{~m}$. The improvement in transmission range and noise immunity could be seen by observing the received signals. To overcome the distortion in the received waveform the signal is passed through a comparator. Fig 7. shows the transmitted and received signals after being corrected by the comparator. From Fig 7. it can be seen that the received signal is identical to the transmitted signal however, there is a slight compensation of delay between them of an amount of 100-500 $\mu$ s, which can be ignored.

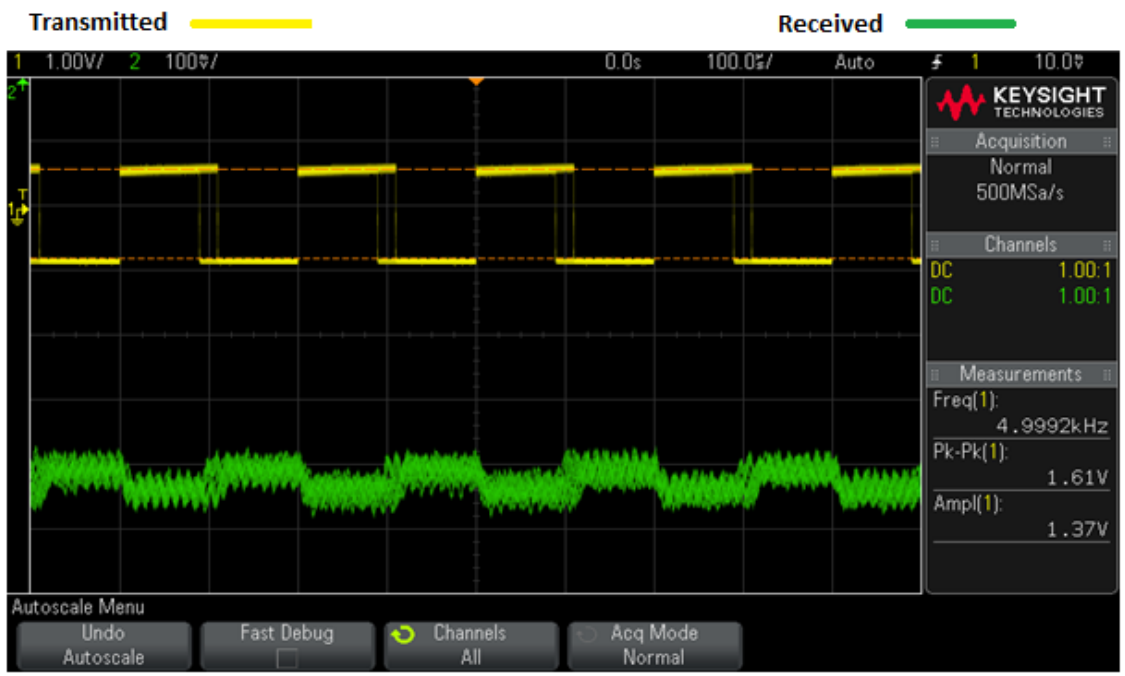

Figure 5. Transmitted and received signal without filtering at distance of $1.5 \mathrm{~m}$.

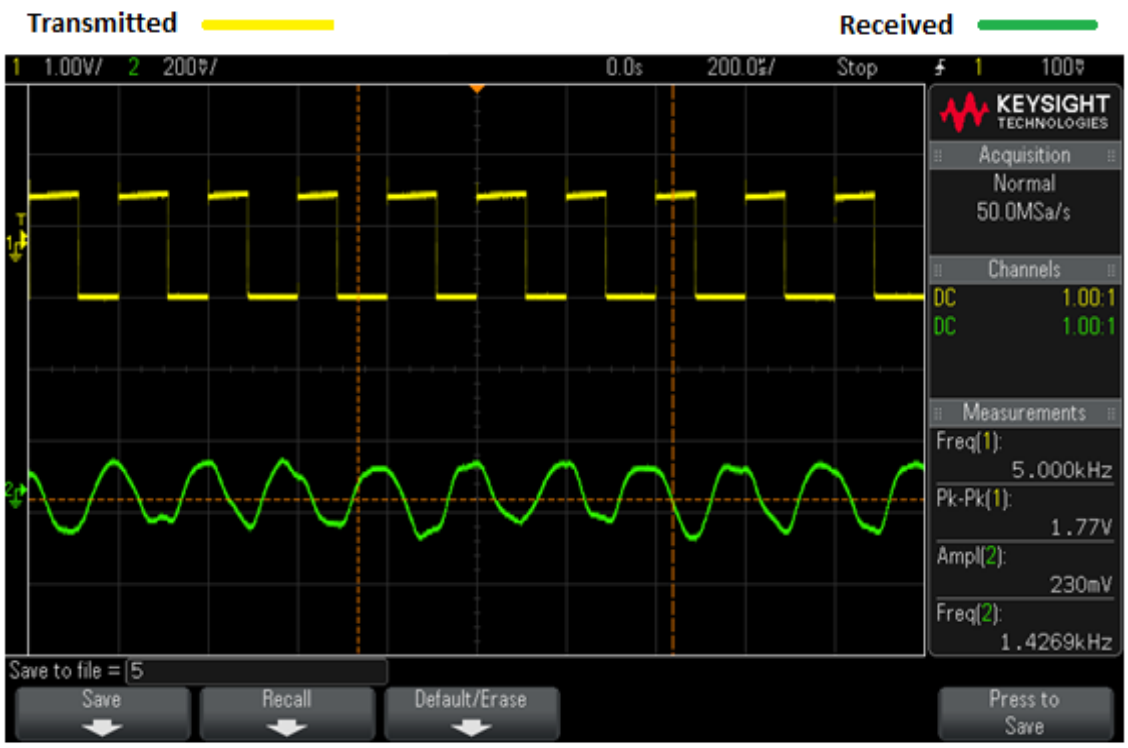

Figure 6. Transmitted and received signal with filtering at distance of $4.7 \mathrm{~m}$. 


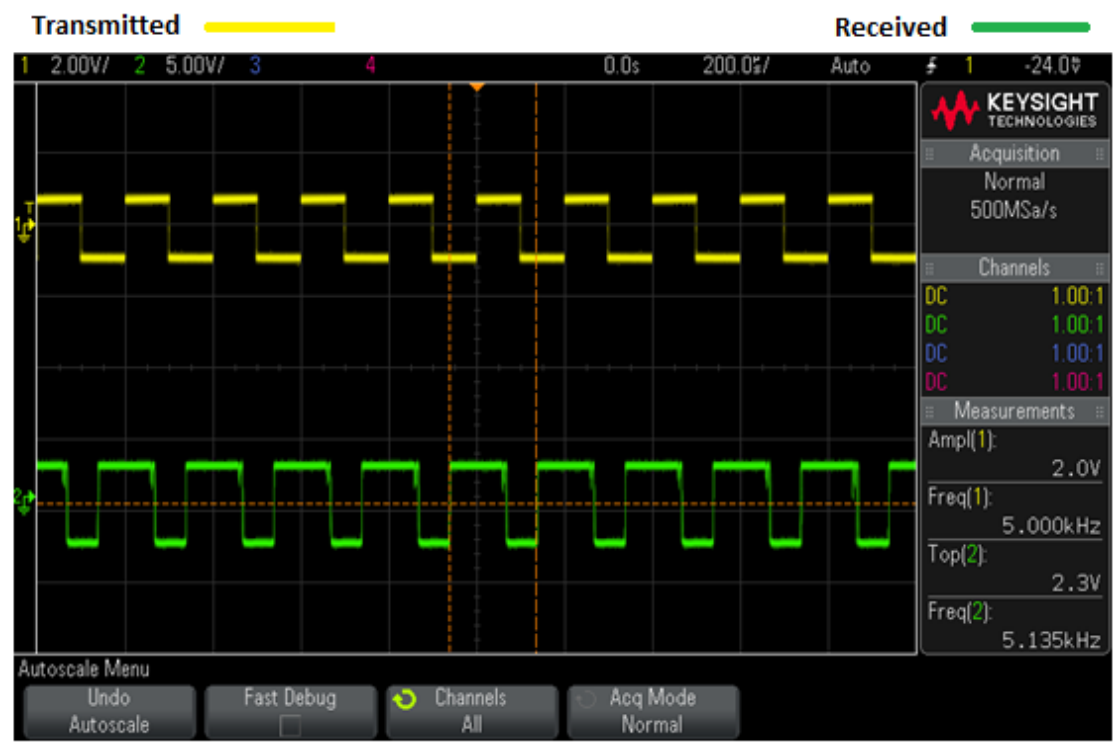

Figure 7. Corrected signal by adding a comparator at distance of $4.7 \mathrm{~m}$.

\subsection{Signal to Noise Ratio (SNR) Vs Distance}

To proof the improvement achieved by employing the proposed MFB band pass filter stage the signal to noise ratio at the output of the MFB stage is evaluated at different distances with and without employing the filtering stage. Fig 8 . shows the signal to noise ratio against distance with and without filtering. As can be observed from the plot, at distance of about 2 meters without filtering, the SNR drops below $1 \mathrm{~dB}$ which means That the signal is covered with noise and can no more be detected, while by employing the proposed MFB filtering stage SNR above $5 \mathrm{~dB}$ is achieved up to a distance of $4 \mathrm{~m}$. By comparing SNR in both cases, we can clearly see that there is significant improvement in SNR over distance with the MFB filtering stage.

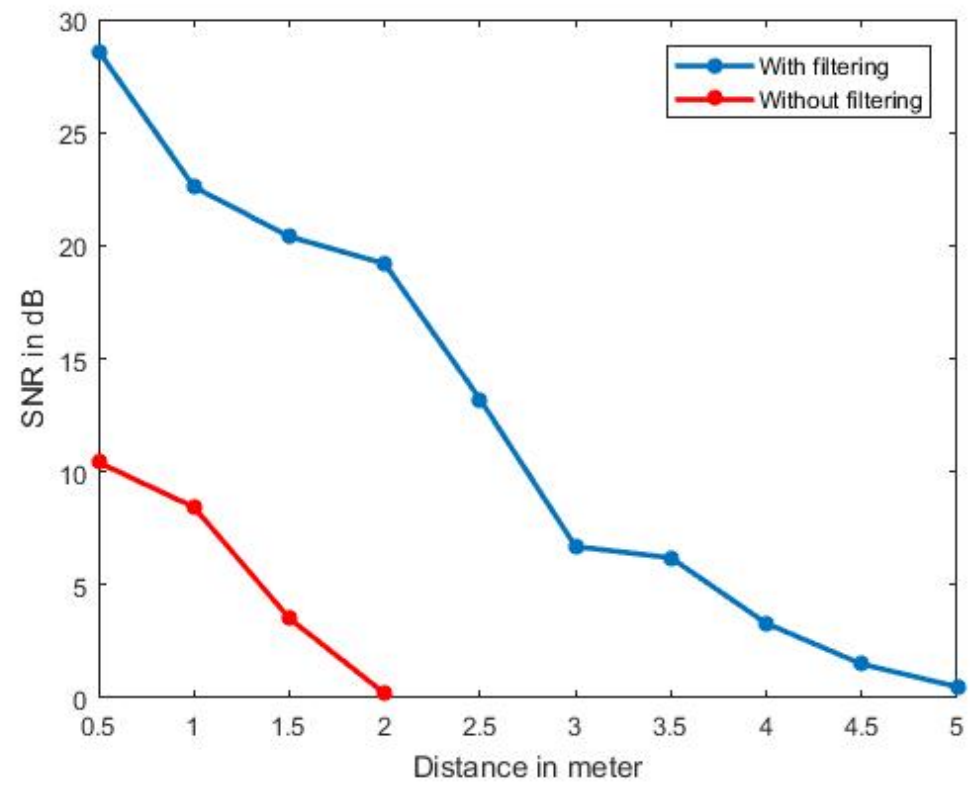

Figure 8. Signal to Noise ratio against distance.

\section{Conclusions}

The aim of this study is to Design and implement a tuned Analog Front-End for extending VLC transmission range. This paper has analysed important factors limiting VLC transmission range and proposed solutions for improving the receiver sensitivity and reducing indoor ambient lights 
interference. By using the proposed MFB filter and an additional comparator stage a long-range VLC system based on PWM was designed and implemented. Experimental results showed that the implemented receiver was not only able to extend the transmission range but can also reduce the effect of fluorescent lights interference. The system performance was measured in terms of SNR over transmission distance. The system was able to achieve a data rate of up to $10 \mathrm{~Kb}$ s over a transmission range of $4.7 \mathrm{~m}$ with a low power white LED for transmission, with the presence of ambient light noise background, however the real BER could not be estimated due to inability of demodulation. Future works should be directed towards applying suitable modulation schemes which could comply with the proposed filtering stage characteristics in order to achieve data rates according to the IEEE 802.15.7 standard. The ability of using high power LEDs to achieve longer-range communication and testing the system performance under the outdoor scenarios should be investigated.

\section{References}

[1] Z. Ghassemlooy, L. Alves, S. Zvánovec and M. Khalighi, Visible light communications: Theory and application, 1st ed. 2017.

[2] M. Basha and R. Binns, "DC biased input stage with differential photocurrent sensing for VLC front-ends", in ICCSS, London, 2017, pp. 167 - 170.

[3] IEEE Standard for Local and Metropolitan Area Networks-Part 15.7: Short-Range Wireless Optical Communication Using Visible Light, IEEE Standard 802.15.7, 2011.

[4] H. Chen, C. Wu, H. Li, X. Chen, Z. Gao, S. Cui and Q. Wang, "Advances and prospects in visible light communications", Journal of Semiconductors, vol. 37, no. 1, p. 011001, 2016.

[5] R. A. Shaikh, A. Basit, “Using point-to-point 1550nm laser for outdoor mobility”, Information and Communication Technologies, 2009. ICICT, pp. 255-258.

[6] F. Chang, W. Hu and D. Lee, "Design and implementation of anti low-frequency noise in visible light communications", in ICASI, Sapporo, 2017, pp. 1536 - 1538.

[7] T. Adiono, A. Pradana and R. Putra, "Analog filters design in VLC analog front-end receiver for reducing indoor ambient light noise", in APCCAS, Jeju, 2016, pp. 581 - 584.

[8] M. Basha, M. Sibley and P. Mather, "Design and implementation of a tuned Analog Front-end for extending VLC transmission range", in IEEE International Conference on Computing, Electronics \& Communications Engineering 2018 (iCCECE '18), 2018, pp. 173-176.

[9] A M Street, P N Stavrinou, D C Obrien and D J Edwards, Indoor optical wireless systems-A review, Optical and Quantum Electronics, 29, 349-378, 1997.

[10] S Hranilovic, On the design of bandwidth efficient signalling for indoor wireless optical channels, International Journal of Communication Systems, 18, 205-228, 2005.

[11] Ghassemlooy, W. Popoola and S. Rajbhandari, Optical wireless communications, 1st ed. Boca Raton, FL: CRC Press, 2013.

[12] Z. Wu, C. You and C. Yang, "Research on long-range real-time visible light communications over phosphorescent LEDs", in CCDC, Chongqing, 2017, pp. 5838 - 5842.

[13] Chung Ghiu Lee, Visible Light Communication, 1st ed. INTECH Open Access Publisher, 2011.

[14] S. Gray, Analysis and Design of Analog Integrated Circuits WIE, 4th Edition, 1st ed. John Wiley \& Sons, 2003.

() 2018 by the author(s). Published by Annals of Emerging Technologies in Computing (AETiC), under the terms and conditions of the Creative Commons Attribution (CC BY) license which can be accessed at http://creativecommons.org/licenses/by/4.0. 